EPJ Web of Conferences 78, 01010 (2014)

DOI: $10.1051 /$ epjconf/20147801010

(C) Owned by the authors, published by EDP Sciences, 2014

\title{
Eugene Wigner - A Gedanken Pioneer of the Second Quantum Revolution
}

\author{
Anton Zeilinger ${ }^{1,2}$ \\ ${ }^{1}$ Faculty of Physics, University of Vienna, Boltzmanngasse 5, 1090 Vienna, Austria \\ ${ }^{2}$ Institute for Quantum Optics and Quantum Information, Austrian Academy of Sciences, \\ Boltzmanngasse 3, 1090 Vienna, Austria
}

\begin{abstract}
Eugene Wigner pointed out very interesting consequences of quantum physics in elegant gedanken experiments. As a result of technical progress, these gedanken experiments have become real experiments and contribute to the development of novel concepts in quantum information science, often called the second quantum revolution.
\end{abstract}

\section{Introduction}

Early quantum mechanics was signified by a very lively debate about its experimental consequences and its conceptual and philosophical implications. In these discussions, gedanken experiments played a significant role. This was because due to experimental limitations at the time, it was not possible to perform experiments with individual quantum systems. That has changed over the years, and now, such experiments have not only been performed, but have become a cornerstone of the second quantum revolution [1].

The first quantum revolution brought explanations of many fundamental phenomena, like atomic physics, solid state physics, magnetism etc., through quantum mechanics. Recently, many of the early gedanken experiments became possible, which opened up a new avenue towards quantum information technology. This is sometimes called "the second quantum revolution".

I had the privilege to personally meet Eugene Wigner at the occasion of the conference "New Techniques and Ideas in Quantum Measurement Theory" which was organized in his honor by Daniel Greenberger in 1986. Wigner not only worked on basic issues in quantum mechanics, but he also had a strong interest in conceptual foundations.

\section{Wigner and superposition and the measurement problem}

In a paper published in American Journal of Physics in 1963 entitled "The Problem of Measurement" [2], Wigner first discusses the gedanken experiment which involves the difference between the superposition of states and statistical mixtures. The essence of his proposal was realized many years later in neutron interferometry [3].

Wigner starts with the quantum state describing both an apparatus and a system:

$$
a \times \sigma^{(v)} \rightarrow a^{(v)} \times \sigma^{(v)}
$$




\section{EPJ Web of Conferences}

Here, the left-hand side describes the initial quantum state of apparatus and system, where $a$ is he initial state of the apparatus and $\sigma$ describes the system state. The system is considered to be in one of its possible eigenstates, in a well-defined state $\sigma^{v}$, with $v$ signifying the specific initial state of the system. Following Wigner, due to the interaction of the apparatus with the system, we arrive at a state where the apparatus is in the specific state $a^{(v)}$. So the complete system is in the product state shown on the right-hand side of Equation (1). So far, nothing surprising has happened.

Yet, Wigner now considers that the system is initially in the superposition state

$$
\sum \alpha_{v} \sigma^{(v)}
$$

where $\alpha_{v}$ are now the amplitudes for the system to be in the specific state $\sigma^{(v)}$. Clearly, such a state is completely legal for the quantum system, and the initial state is again the product state of the apparatus $a$ with now the superposition as shown on the left-hand side of Equation (3):

$$
\mathrm{a} \times\left[\sum \alpha_{v} \sigma^{(v)}\right] \rightarrow \sum \alpha_{v}\left[\mathrm{a}^{(v)} \times \sigma^{(v)}\right]
$$

Again following Wigner, due to interaction between the apparatus and the system, the state on the right-hand side is obtained where $a^{(v)}$ is now a specific apparatus state corresponding to the specific system state $\sigma^{(v)}$. Clearly, that state is a highly entangled quantum state, with neither apparatus nor system enjoying a well-defined specific state on their own. But, as Wigner argues, observation of that state yields a specific result. Therefore the final state now is

$$
a^{(\mu)} \times \sigma^{(\mu)}
$$

Certainly, that state is just a product state corresponding to the specific result obtained. As Wigner shows, the transition from the entangled state to the specific output state cannot be described within quantum mechanics.

Interestingly, the realization of that gedanken experiment had to wait until 1983, when it was performed in the group of Helmut Rauch in Vienna [3] with a neutron interferometer. Fig. 2 shows the experimental setup:

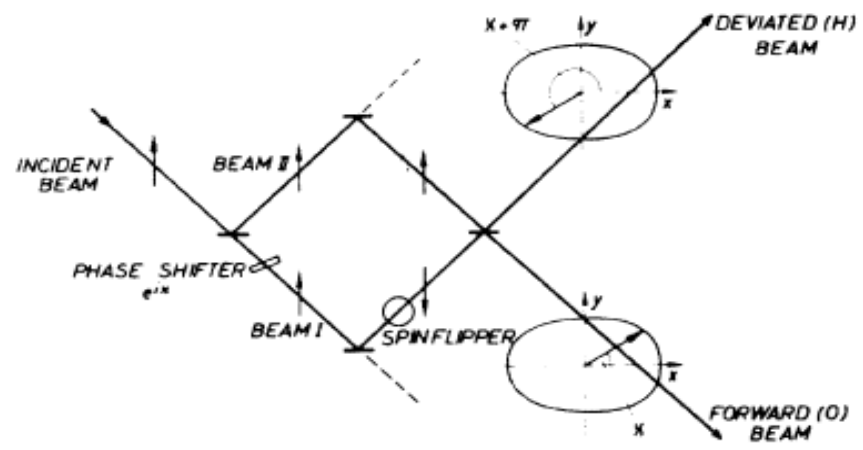

Figure 1. Experimental test of spin superposition. The two output beams of a neutron interferometer are equalamplitude superpositions of orthogonal spin-up and spin-down states and therefore lie in the horizontal plane. The specific angle of the polarization vectors can be rotated by a nuclear phase shift inside the interferometer. Also, not shown here, a measurement of spin along the vertical axis leads up or down, which corresponds to the specific result obtained (from [3]).

The idea of the experiment is rather straightforward. We start with a neutron beam, which is spinup, and then split it into two beams, beam I and beam II. Then with a spin flipper, the spin in beam I is flipped to pointing downwards. This is exactly the situation in the Wigner gedanken experiment. When in the final beam splitter the two spins are superposed, according to orthodox theory (following Wigner's naming), the resulting spin should then point horizontally. 
It turns out that the two emerging beams, the forward beam and the deviated beam, after the interferometer both have horizontally pointing spins, but rotated with respect to each other by $\pi$. This is a consequence of the unitary operation of the interferometer. In addition with the phase shifter put into one of the two beams, one can rotate both beams in the $x-y$ plane.

The experiment completely confirmed that the spins were pointing in the horizontal direction. Therefore, the output beams cannot be considered to be just a statistical mixture. This experiment transformed Wigner's gedanken experiment into the realm of reality and underlines the significant difference between a coherent superposition of orthogonal states and a statistical mixture. It should be noted that by now, this phenomenon has become a standard in many experiments performed with many kinds of quantum states, for example also atoms or ions, in many laboratories world-wide.

\section{Wigner and Einstein-Podolsky-Rosen}

In the same paper of 1963 [2], Wigner considered an Einstein-Podolsky-Rosen type state [4]. As is well known, that state is entangled both in position and in momentum. Again, the question is whether, as prescribed by quantum mechanics, the state has to be seen as a superposition of momentum (or position) states of the two particles, or if it would be sufficient to just see it as a statistical mixture. Wigner writes:

"It would be wrong ... to say that even before any measurement, the state was a mixture of plane waves of the two particles, travelling in opposite directions. For no such pair of plane waves would one expect the correlation of angular momentum just described."

A most recent confirmation of the fact that in such an entangled state, one does not have a mixture of the individual plane waves, was an experiment where entanglement of high orbital angular momenta was obtained [5]. In that experiment, one converts the polarization state of two photons

$$
\frac{1}{\sqrt{2}}(|H, V\rangle+|V, H\rangle)
$$

using spatial light modulators into the orbital angular momentum entangled state

$$
\frac{1}{\sqrt{2}}(|1,-1\rangle+|-1,1\rangle)
$$

where $l$ is the topological charge of an orbital angular momentum state [6]. In the experiment, it was possible to project the orbital angular momentum states onto superpositions of $+l$ and $-l$, with topological charges up to 300 . Orbital angular momentum states can be seen as superpositions of many plane waves.

Thus our result confirms that we observe entanglements between rather intricate superpositions of plane waves. The observed high-visibility entanglement would clearly not have been possible if the states had just been mixtures of plane waves of the two photons.

\section{Wigner and Bell's Theorem}

Wigner was also one of the first physicists interested in Bell's theorem. In a paper entitled "On Hidden Variables and Quantum Mechanical Probabilities" published in 1970 in the American Journal of Physics [7] he provided some fundamental insights and also a novel proposal for an experiment. In that paper, Wigner considers the entanglement of two spin- $1 / 2$ particles just like John Bell did [8], but discovers that correlations between three measurement directions would lead to a more intuitive argument against hidden variables.

The question Wigner raises is whether the following equation can be true: 


$$
\begin{aligned}
& \sum \sum_{\sigma_{2} \sigma_{3} \tau_{1} \tau_{2}}\left(+\sigma_{2}, \sigma_{3} ; \tau_{1}, \tau_{2}+\right) \\
& =(++-;--+)+(+--;-++) \\
& =\frac{1}{2} \sin ^{2} \frac{1}{2} \vartheta_{31} .
\end{aligned}
$$

Here in the top line, we have the states expressed in hidden variables, $\sigma$ for particle 1 and $\tau$ for particle 2. The three entries correspond to three possible directions of a Stern-Gerlach magnet. For example, $\sigma_{2}$ would indicate a result of +1 or -1 for the first particle along the chosen direction 2 , and $\tau_{2}$ would correspond to the result +1 or -1 along the same direction 2 for the second particle.

For the specific case of considering + results for the first particle along some direction 1 and + results for the second particle along some other direction 3, one has to sum over the unseen results along the here unmeasured direction 2 if one considers a hidden variable model. Thus, the remaining terms in the sum can just be the two terms shown in the second line of the equation above. Then, quantum mechanics prescribes that the correlations between two arbitrary direction settings for the first particle and the second particle should follow the sign squared rule as given in the third line, where $\vartheta_{31}$ is the angle between the two measurement directions 1 and 3 . Wigner then shows that this equation cannot be fulfilled for all arbitrary directions and arrives at a new inequality, which improves the original Bell inequality from a conceptual point of view.

As Wigner writes, the conclusion is that his argument

"... shows that a description of the quantum mechanical measurement of the spin components of these two particles by means of hidden parameters is impossible if we assume that the parameters determining the outcome of the measurement of the spin of each particle are independent of the direction in which we decide to measure the spin of the other particle."

There have been a number of experiments aiming at showing that the violation of this independence cannot be due to some unknown communication between the two measurement setups or between the two particles. The situation is most striking if one considers entanglement swapping [9], that is, the teleportation of an entangled state [10]. Most significantly, Asher Peres has added an interesting twist to the discussion by suggesting delayed-choice entanglement swapping, that is, delayed teleportation of an entangled state [11].

The experiment proceeds as follows: One starts with two entangled pairs from EPR Source I and EPR Source II. Let us assume for simplicity that these are photons entangled in polarization. Then, entanglement swapping in principle is the idea to project one photon from each pair ( 2 and 3 in Figure 2) onto an entangled state, and therefore the entanglement is transferred to the other two photons 1 and 4 of each entangled pair. Thus, photons 1 and 4 that have never interacted or have not come from a common source become entangled. The additional point suggested by Peres is to measure the two outer photons at some earlier time and then, at some later time, after the outer photons have been registered, to decide whether photons 2 and 3 (Figure 2) are projected onto an entangled state or measured separately. Thus, at a later state, one can decide whether the already registered photons 1 and 4 were entangled or not.

It is certainly most interesting to delay the choice of whether photons 2 and 3 are projected onto the entangled state or not to a time after the other two photons have already been registered. Such an experiment was realized by Ma et al. [12].

In the experiment, two pairs of photons, 1-2 and 3-4, were created by parametric down-conversion. Photon 1 and photon 4 were subjected immediately to polarization measurements, and the results were recorded. Photons 2 and 3 were delayed in a glass fiber and then sent into a Mach Zehnder interferometer. In the Mach Zehnder interferometer, the final outgoing beams can be superpositions of the two inputs, or they can correlate perfectly with the inputs depending on the internal phase. If the internal phase of the interferometer is a multiple of $\pi$, then there is a one to one correspondence between one of the two input ports and one of the two output ports. Therefore, measuring of the 
photons in the output provides a measurement of the polarization of the individual photons. On the other hand, if the internal phase is $\frac{\pi}{2}$ (modulo $\pi$ ), then each output is a superposition of the two inputs.

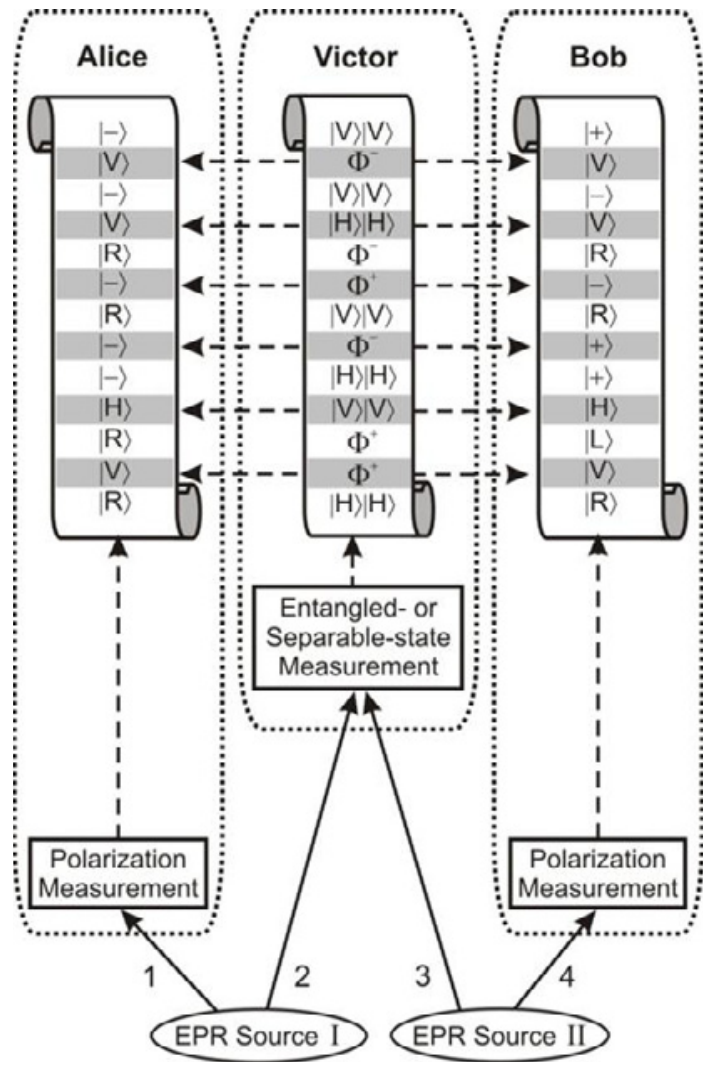

Figure 2. The concept of delayed-choice entanglement swapping. Two entangled pairs of photons $1 \& 2$ and $3 \& 4$ are produced in the state $\left|\psi^{-}\right\rangle_{12} \times\left|\psi^{-}\right\rangle_{34}$ in the EPR sources I and II, respectively. At first, Alice and Bob perform polarization measurements on photons 1 and 4, choosing freely the polarization analysis basis among three mutually unbiased bases $(|H\rangle /|V\rangle,|R\rangle /|L\rangle,|+\rangle /|-\rangle)$, and record the outcomes. Photons 2 and 3 are sent to Victor, who then subjects them to either an entangled or a separable-state measurement, projecting them randomly onto one of two possible Bell states $\left(\left|\Phi^{+}\right\rangle_{23}\right.$ or $\left.\left|\Phi^{-}\right\rangle_{23}\right)$ or one of two separable states $\left(|H H\rangle_{23}\right.$ or $\left.|V V\rangle_{23}\right)$. Victor records the outcome and keeps it to himself. This procedure projects photons 1 and 4 onto a corresponding entangled $\left(\left|\Phi^{+}\right\rangle_{14}\right.$ or $\left.\left|\Phi^{-}\right\rangle_{14}\right)$ or separable state $\left(|V V\rangle_{14}\right.$ or $\left.|H H\rangle_{14}\right)$, respectively. According to Victor's choice and his results, Alice and Bob can sort their already recorded data into subsets and can verify that each subset behaves as if it consisted of either entangled or separable pairs of distant photons, which have neither communicated nor interacted in the past (from [12]).

Thus, in that case, if we have two input photons and register one photon in each output, the two incoming photons are projected onto the antisymmetric entangled state $\psi^{-}$and, if the two photons are registered with different polarizations but in the same output, the incoming state is projected into the entangled state $\psi^{+}[13,14]$. In the experiment, the internal phase was set in the last instant using electro-optical modulators driven by a rapid quantum random number generator.

As the experimental results clearly demonstrate, the two already registered photons are entangled if the two inner photons are projected onto an entangled state, and they are not entangled if they are measured individually. This is confirmed by using a special entanglement witness, which in essence consists in observing the correlations in three mutually unbiased bases. The experiment may also be seen as a confirmation of Wigner's view presented above. 

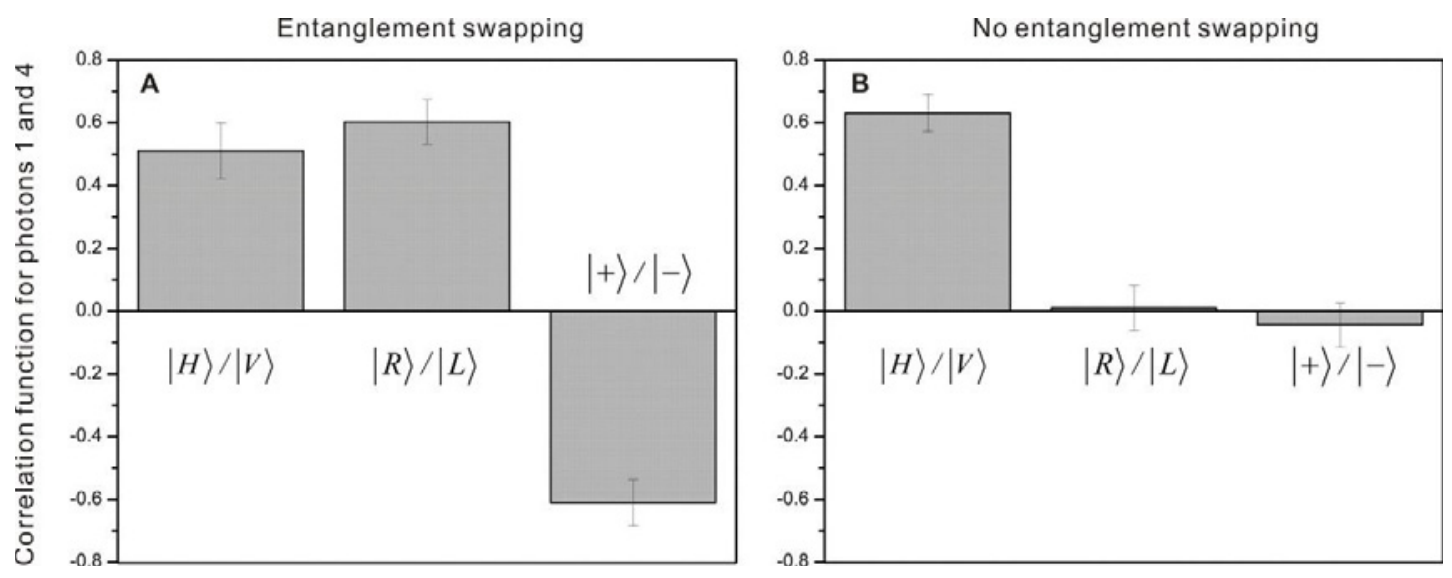

Figure 3. Experimental results of the delayed-choice entanglement swapping experiment: correlation function between photons 1 and 4 for the three mutually unbiased bases $(|H\rangle|V\rangle,|R\rangle|L\rangle,|+\rangle|-\rangle)$. Victor subjects photons 2 and 3 to either (A) an entangled or (B) a separable-state measurement. These results are obtained via coincidence counts of photons 1 and 4 , conditioned on the coincidence of same polarization and different spatial output modes of photons 2 and 3 ( $b^{\prime \prime}$ and $c^{\prime \prime}$ in Fig. 2). (A) When Victor performs a Bell-state measurement and finds photons 2 and 3 in the state $\Phi-\rangle_{23}=\frac{\left.(H H\rangle_{23}-|V V\rangle_{23}\right)}{\sqrt{2}}$, entanglement is swapped to photons 1 and 4 . This is confirmed by all three correlation functions being of equal magnitude (within statistical error) and their absolute sum exceeding 1. (B) When Victor performs a separable-state measurement and finds photons 2 and 3 in either the state $|\mathrm{HH}\rangle_{23}$ or $-|V V\rangle_{23}$, entanglement is not swapped. This is confirmed by only the correlation function in the $|H\rangle|V\rangle$ basis being significant while the others vanish. The experimentally obtained correlation functions of photons 1 and 4 in the $|H\rangle|V\rangle,|R\rangle|L\rangle,|+\rangle|-\rangle$ bases are $0.511 \pm 0.089,0.603 \pm 0.071,-0.611 \pm 0.074$ respectively for case (A) and $0.632 \pm 0.059,0.01 \pm 0.072,-0.045 \pm 0.070$ respectively for case (B). While entangled states can show maximal correlations in all three bases (the magnitude of all correlation functions equals 1 ideally), separable states can be maximally correlated (ideal correlation function 1) only in one basis, the others being 0 . The uncertainties represent plus/minus one standard deviation deduced from propagated Poissonian statistics (from [12]).

\section{Conclusion}

It would be fascinating to know Eugene Wigner's reaction to the fact that the gedanken experiments he discussed have not only become reality, but building on his gedanken experiments, new ideas have developed which on the one hand probe the foundations of quantum mechanics even deeper, and which on the other hand also provide the foundations to the new field of quantum information technology. All these experiments pay homage to the great insight Wigner expressed in developing these gedanken experiments and in his analyses of the foundations of quantum mechanics, as for example also presented in his 1976 talk at Eötvös University [15].

\section{Acknowledgments}

I would like to acknowledge the great hospitality of Professor Sándor Varró and his team at the occasion of the symposium "Wigner 111 - Colourful \& Deep" which took place in 2013 in Budapest. The work presented here was supported by many institutions, including the European Research Council and the Austrian Science Fund. 


\section{References}

1. J.P. Dowling, G.J. Milburn, Phil. Trans. R. Soc. A 361, 3655 (2003)

2. E.P. Wigner, Amer. J. Phys. 31 (1), 6 (1963)

3. J. Summhammer, G. Badurek, H. Rauch, U. Kischko, A. Zeilinger, Phys. Rev. A 27, 2523 (1983)

4. A. Einstein, B. Podolsky, N. Rosen, Phys. Rev. 47, 777 (1935)

5. R. Fickler, R. Lapkiewicz, W.N. Plick, M. Krenn, C. Schaeff, S. Ramelow, A. Zeilinger, Science 338 (6107), 640 (2012)

6. L. Allen, M.W. Beyersbergen, R.J.C. Spreeuw, J.P.. Woerdman, Phys. Rev. A 45, 8185 (1992)

7. E.P. Wigner, Am. J. Phys. 38, 1005 (1970)

8. J. Bell, Physics 1 (3), 195 (1964).

9. M. Zukowski, A. Zeilinger, M.A. Horne, A.K. Ekert, Phys. Rev. Lett. 71, 4287 (1993)

10. C.H. Bennett, G. Brassard, C. Crépeau, R. Jozsa, A. Peres, W.K. Wootters, Phys. Rev. Lett. 70, 1895 (1993)

11. A. Peres, J. Mod. Opt. 47, 139 (2000)

12. X.-S. Ma, S. Zotter, J. Kofler, R. Ursin, T. Jennewein, Č. Brukner, A. Zeilinger, Nature Physics 8, 480 (2012)

13. H. Weinfurter, Europhys. Lett. 25, 559 (1994)

14. M. Michler, K. Mattle, H. Weinfurter, A. Zeilinger, Phys. Rev. A 53 (3), R1209 (1996)

15. E.P. Wigner, Fizikai Szemle 27, 81 (1977) 
